Proceedings

\title{
Postprandial Bioactivity of Spread Cheese, Enhanced with Mountain Tea and Orange Peel Extract, in Healthy Volunteers. A Pilot Study ${ }^{\dagger}$
}

\author{
Olga Papagianni ${ }^{1}$, Thomas Loukas ${ }^{2}$, Athanasios Magkoutis ${ }^{2}$, Theodora Biagki ${ }^{1}$, Charalampia Dimou ${ }^{1}$, \\ Charalampos Karantonis ${ }^{3}$ and Antonios Koutelidakis ${ }^{1, *}$
}

Citation: Papagianni, O.; Loukas, T.; Magkoutis, A.; Biagki, T.; Dimou, C.; Karantonis, C.; Koutelidakis, A. Post-prandial Bioactivity of Spread Cheese, Enhanced with Mountain Tea and Orange Peel Extract, in Healthy Volunteers. A Pilot Study. Proceedings 2021, 70, 19. https://doi.org/ 10.3390/foods_2020-07671

Published: 9 November 2020

Publisher's Note: MDPI stays neutral with regard to jurisdictional claims in published maps and institutional affiliations.

Copyright: $\odot 2020$ by the authors. Licensee MDPI, Basel, Switzerland. This article is an open access article distributed under the terms and conditions of the Creative Commons Attribution (CC BY) license (http://creativecommons.org/licenses/by/4.0/).
1 Laboratory of Nutrition and Public Health, Human Nutrition Unit, Food Science and Nutrition Department, University of the Aegean, 11472 Myrina, Limnos, Greece; olga3_pap@yahoo.gr (O.P.); fns15064@fns.aegean.gr (T.B.); chadim@aegean.gr (C.D.)

2 Outpattent Clinic, 11472 Myrina, Limnos, Greece; tloukas2002@yahoo.com (T.L.); tmagoutis@gmail.com (A.M.)

3 Laboratory of Food Chemistry, Biochemistry and Technology, Food Science and Nutrition Department, University of the Aegean, 11472 Myrina, Limnos, Greece; chkarantonis@aegean.gr

* Correspondence: akoutel@aegean.gr

+ Presented at the 1st International Electronic Conference on Food Science and Functional Foods, 10-25 November 2020; Available online: https://foods_2020.sciforum.net/.

\begin{abstract}
Post-prandial lipemia, glycemia and oxidative stress may affect the outcome of cardiovascular disease. It has been investigated that the enhancement of spread cheese with mountain tea (Sideritis sp.) and orange peel extract, may reduce post-prandial metabolic biomarkers in healthy volunteers. The purpose of the present pilot study was to investigate the possible post-prandial bioactivity of such a spread cheese. In the framework of cross-over design, nine healthy volunteers 20-30 years old, consumed a meal, rich in fat and carbohydrates ( $80 \mathrm{~g}$ white bread, $40 \mathrm{~g}$ butter and $30 \mathrm{~g}$ full fat spread cheese). After a week washout period, the same volunteers consumed the same meal with the spread cheese, enhanced with $6 \%$ mountain tea-dried orange peel extract. Blood sampling took place before, 1.5, 3 and $5 \mathrm{~h}$ after meal consumption. Total plasma antioxidant capacity, serum lipids, glucose, uric acid and anticoagulant activity were measured at each time point. There was a statistically significant increase in the antioxidant capacity of plasma $3 \mathrm{~h}$ after the meal consumption in the presence of cheese enhanced with extract, compared to the consumption of conventional cheese $(p<0.05)$. The increase rate of glucose and triglycerides showed a decreasing tendency, $1.5 \mathrm{~h}$ after eating the meal with the extract. The remaining biomarkers did not show statistically significant differences $(p>0.05)$. More studies in a larger sample are needed to draw safer conclusions about the effect of extract on metabolic biomarkers, such as oxidative stress, lipemia and glycemia.
\end{abstract}

Keywords: post-prandial bioactivity; bioactive compounds enhanced cheese; orange peel extract; mountain tea extract; metabolic biomarkers

\section{Introduction}

The dynamic period, which includes metabolic processes after digestion and absorption, is described as the post-prandial state. Prolonged and excessive metabolic (lipemia, glycemia) and oxidative imbalance that can occur after a meal is called "post-prandial oxidative stress" [1]. Scientific evidence suggests that post-prandial lipemia, glycemia and oxidative stress may promote cell dysfunction and affect the outcome of cardiovascular disease (CVD) $[2,3]$. The sudden increase in plasma glucose and triglycerides, after consuming a meal is reported to be an activator of endothelial cell inflammation. Furthermore, oxidative stress is a promotive factor in the effect of metabolic stress on vascular 
dysfunction and the formation of inflammation. Macronutrients in the consumed meal influence the post-prandial responses [4].

The term "functional foods" first appeared in Japan when, in 1984, the country's scientific community defined functional foods as performing three basic functions: meeting nutritional needs, providing satisfaction and having a beneficial effect on the human body. By "functional" we mean foods, processed or not, which have been proven-based on scientific studies - that due to their bioactive constituents they contribute to the achievement of specific functional goals within the body, contributing to health promotion. Examples of functional ingredients in foods are carotenoids, probiotics and polyphenols [5]. It has been reported that mountain tea (Sideritis sp.) and orange peel (fruit byproduct), as natural functional foods, contain bioactive compounds with possible beneficial effects on serum lipids and glucose levels, as well as on plasma total antioxidant activity [6,7].

Given the fact that the consumption of a meal, rich in fat and carbohydrates, could promote oxidative stress and post-prandial lipemia and glycaemia, the enhancement of a spread cheese with natural bio-functional foods, such as mountain tea (Sideritis sp.) and orange peel extract, with the aim to reduce post-prandial metabolic biomarkers in healthy volunteers has been investigated.

The purpose of this study was to investigate the possible post-prandial bioactivity of a spread cheese, enhanced with mountain tea-orange peel extract.

\section{Materials and Methods}

In this randomized, cross-over design study, pilot, intervention study, 9 healthy volunteers 20-30 years old, consumed a meal rich in fat and carbohydrates, ( 2 slices $80 \mathrm{~g}$ of white bread, spread with $40 \mathrm{~g}$ of butter and $30 \mathrm{~g}$ of full fat spread cheese (control meal). After a week washout period, the same volunteers consumed the same meal contained instead of the control cheese, a novel spread cheese enhanced with $6 \%$ mountain tea and dried orange peel extract (intervention meal). Blood sampling was performed, before and $1.5,3$ and $5 \mathrm{~h}$ after the meal. The total antioxidant capacity (FRAP assay) [3], serum lipids (Total, High Density Lipoprotein (HDL), Low Density Lipoprotein (LDL)-cholesterol and triglycerides), glucose and uric acid were measured using a Roche Cobas c111 biochemical analyzer, while anticoagulant activity were also determined for each time point [7].

The study protocol was approved by the Ethics committee of University of the Aegean and the study was performed in accordance with the Declaration of Helsinki. All the participants signed an informed consent form and were informed about the prime target of this study, the confidentiality of data and the voluntary nature of participation. All participants were initially screened by using a medical history questionnaire that also included demographic characteristics, level of physical activity and consumption of foods rich in polyphenols in the preceding period. Furthermore, anthropometric measurements took place.

\section{Preparation of the Novel Spread Cheese}

The extract was prepared by adding $3 \mathrm{~g}$ of mountain tea and $3 \mathrm{~g}$ of dried orange peel to $100 \mathrm{~mL}$ of boiling water, and then boiling for $5 \mathrm{~min}$ to extract the bioactive compounds. Filtration of the extract was followed by filter paper. The mountain tea-orange peel extract contained $49.9 \mu \mathrm{g}$ gallic acid/mL total phenolics (Folin-ciocalteu method) and had a total antioxidant capacity of $34.67 \mu \mathrm{mol} \mathrm{Fe}_{2} \mathrm{SO}_{4} / \mathrm{mL}$ (FRAP method). The functional spread cheese was prepared adding $6 \mathrm{~mL}$ of mountain tea-orange peel extract to $94 \mathrm{~g}$ cheese, followed by mixture homogenization.

\section{Results and Discussion}

The basic result of the study was that a statistically significant increase was observed in the total antioxidant capacity of plasma $3 \mathrm{~h}$ after the consumption of the intervention 
meal (enhanced cheese with mountain tea-orange peel extract), compared to the consumption of the control meal (control cheese) $(p<0.05)$ (see Figure 1). Furthermore, there was a decrease in the tendency of the glucose and triglycerides increase rate $1.5 \mathrm{~h}$ after the consumption of the intervention meal. The remaining biomarkers did not show statistically significant differences $(p>0.05)$.

These findings suggest that the enhancement of a spread cheese with mountain tea and orange peel extract may be beneficial on the antioxidant capacity of the plasma, attenuating possible post-prandial oxidative stress. The phenolic compound metabolites may also affect the post-prandial levels of serum triglycerides and glucose levels. Nevertheless, this was a pilot study; thus the extension of the study with more participants could lead to safer conclusions.

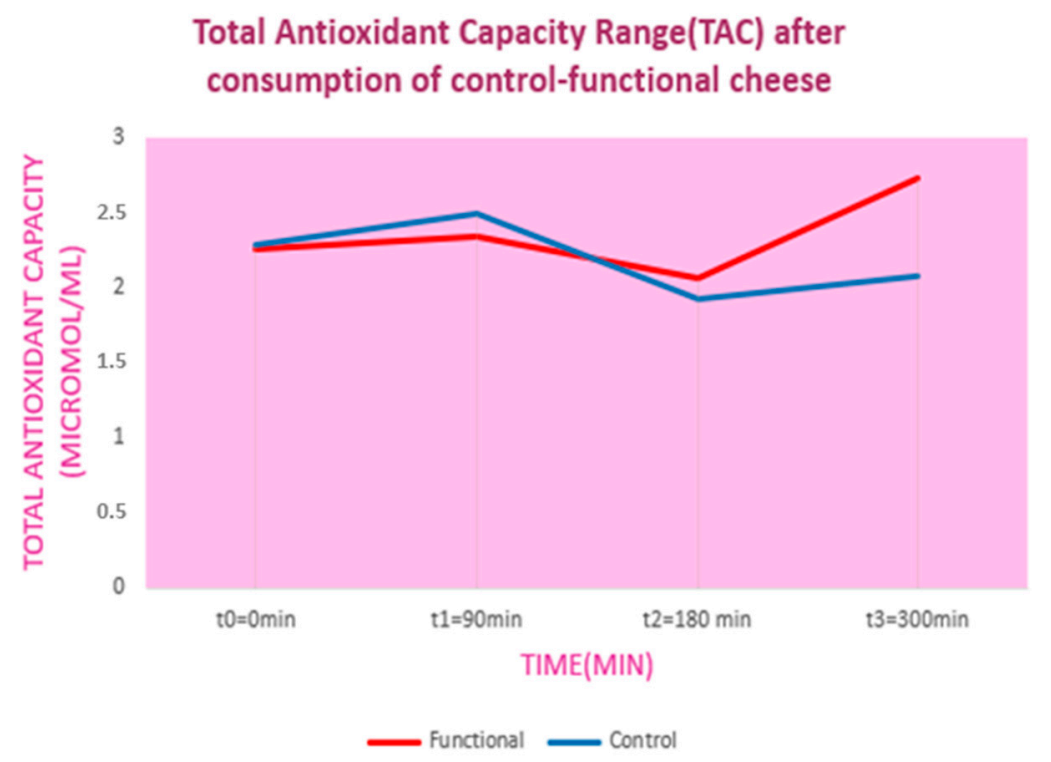

Figure 1. Value range curves for total antioxidant capacity of volunteer plasma, after consuming control and functional, spreadable cheese, respectively.

\section{Conclusions}

The present study showed a possible effect of natural bioactive compounds on postprandial plasma antioxidant capacity. Clinical trials are continuing and expanding in a larger sample of the population, in order to draw safer conclusions about the effect of the extraction of post-prandial biomarkers of oxidative stress, lipemia and glycemia, factors that significantly affect the risk of cardiovascular disease (CVD).

Funding: This research funded from the research program TYRELAIA. Development of functional spreadable cheeses enriched with extracts and essential oils of aromatic and medicinal plants from Western Greece, ESPA, BUSINESS PROGRAM WESTERN GREECE 2014-2020.

Institutional Review Board Statement: The study was conducted according to the guidelines of the Declaration of Helsinki, and approved by the Ethics Committee of University of the Aegean (Oct 2019).

Informed Consent Statement: Informed consent was obtained from all subjects involved in the study.

Data Availability Statement: Not applicable.

Conflicts of Interest: The authors declare no conflict of interest. 


\section{References}

1. Diekmann, C.; Huber, H.; Preu, M.; Preu, P.; Predel, H.D.; Stoffel-Wagner, B.; Fimmers, R.; Stehle, P.; Egert, S. Moderate Postmeal Walking Has No Beneficial Effects Over Resting on Postprandial Lipemia, Glycemia, Insulinemia, and Selected Oxidative and Inflammatory Parameters in Older Adults with a Cardiovascular Disease Risk Phenotype: A Randomized Crossover Trial. J. Nutr. 2019, 149, 1930-1941.

2. Le, N.A. Postprandial Triglycerides, Oxidative Stress, and Inflammation in Triglycerides and Cholesterol. Available online: https://www.intechopen.com/books/apolipoproteins-triglycerides-and-cholesterol/postprandial-triglycerides-oxidative-stressand-inflammation (accessed on 25 June 2020).

3. Koutelidakis, A.E.; Rallidis, L.; Koniari, K.; Panagiotakos, D.; Komaitis, M.; Zampelas, A.; Anastasiou-Nana, M.; Kapsokefalou, M. Effect of green tea on postprandial antioxidant capacity, serum lipids, C Reactive Protein and glucose levels in patients with coronary artery disease. Eur. J. Nutr. 2013, 53, 479-486.

4. Dimina, L.; Mariotti, F. The Postprandial Appearance of Features of Cardiometabolic Risk: Acute Induction and Prevention by Nutrients and Other Dietary Substances. Nutrients 2019, 11, 1963.

5. Konstantinidi, M.; Koutelidakis, A.E. Functional Foods and Bioactive Compounds: A Review of Its Possible Role on Weight Management and Obesity's Metabolic Consequences. Medicines 2019, 6, 94.

6. Dimou, C.; Karantonis, C.; Skalkos, D.; Koutelidakis, A.E. Valorization of fruits by-products to unconventional sources of additives, oil, biomolecules and innovative functional foods. Curr. Pharm. Biotechnol. 2019, 20, 776-786.

7. Georgakopoulou, V.; Dimou, C.; Karantonis, C. In Vitro Antioxidant, Antithrombotic, Antiatherogenic and Antidiabetic Activities of Urtica dioica, Sideritis euboea and Cistus creticus Water Extracts and Investigation of Pasta Fortification with the Most Bioactive One. Curr. Pharm. Biotechnol. 2019, 20, 874-880. 